\title{
Optimization of Bayesian Emission Tomographic Reconstruction for Region of Interest Quantitation
}

\author{
Jinyi Qi \\ Lawrence Berkeley National Laboratory, One Cyclotron Road, Berkeley, CA 94720, USA
}

\begin{abstract}
Region of interest (ROI) quantitation is an important task in emission tomography (e.g., positron emission tomography and single photon emission computed tomography). It is essential for exploring clinical factors such as tumor activity, growth rate, and the efficacy of therapeutic interventions. Bayesian methods based on the maximum a posteriori principle (or called penalized maximum likelihood methods) have been developed for emission image reconstructions to deal with the low signal to noise ratio of the emission data. Similar to the filter cut-off frequency in the filtered backprojection method, the smoothing parameter of the image prior in Bayesian reconstruction controls the resolution and noise trade-off and hence affects ROI quantitation. In this paper we present an approach for choosing the optimum smoothing parameter in Bayesian reconstruction for ROI quantitation. Bayesian reconstructions are difficult to analyze because the resolution and noise properties are nonlinear and object-dependent. Building on the recent progress on deriving the approximate expressions for the local impulse response function and the covariance matrix, we derived simplified theoretical expressions for the bias, the variance, and the ensemble mean squared error (EMSE) of the ROI quantitation. One problem in evaluating ROI quantitation is that the truth is often required for calculating the bias. This is overcome by using ensemble distribution of the activity inside the ROI and computing the average EMSE. The resulting expressions allow fast evaluation of the image quality for different smoothing parameters. The optimum smoothing parameter of the image prior can then be selected to minimize the EMSE.
\end{abstract}

Keywords: Emission tomography, quantitation, MAP reconstruction, parameter estimation

\section{INTRODUCTION}

Emission tomography using radioactive tracers (namely, single photon emission computed tomography [SPECT] and positron emission tomography $[\mathrm{PET}]$ ) has been widely used in clinical diagnosis and biological research. One important application of emission tomography is to quantify the absolute tracer uptake in regions of interest (ROIs). ROI quantitation is very important for evaluating tumor activity, growth rate, and efficacy of therapeutic interventions.

People have studied the quantitative results of emission reconstruction. However, these studies have mostly been focused on linear algorithms. Statistical methods have been developed for emission tomography to improve image quality. ${ }^{1-4}$ However, because they are generally nonlinear, the analysis is more difficult. Recently, progress has been made in understanding the nonlinear properties of statistical reconstruction methods. ${ }^{5-11}$

Barrett et al. ${ }^{5}$ derived approximate formulae for the mean and covariance of the maximum likelihood (ML) expectation maximization (EM) reconstruction as a function of the iteration number. The same approach was extended to maximum a posteriori (MAP) EM algorithms by Wang and Gindi ${ }^{8}$ and most recently to block iterative algorithms by Soares et al. ${ }^{12}$ This iteration-based approach is attractive for methods that are terminated before convergence, as is common practice for the EM algorithm and its ordered-subsets variants. ${ }^{13}$ However, evaluation of the expressions for large numbers of iterations is time-consuming. In addition, this approach requires that the reconstruction algorithm has an explicit update equation. Hence, it is not applicable to gradient-type algorithms that involve line searches.

An alternative approach was proposed by Fessler and Rogers, ${ }^{6,7}$ who analyzed the mean, variance, and spatial resolution at a fixed point of the objective function. The resolution and noise properties are computed at the fixed

Further author information: E-mail: jqi@lbl.gov, Telephone: 15104864695 
point using partial derivatives and truncated Taylor series approximations. These results are independent of the particular optimizing algorithm used and require only that the algorithm be iterated to effective convergence. Qi and Leahy ${ }^{9,10}$ extended this approach by deriving simplified expressions for the local impulse response function and covariance using Fourier transform. These expressions allow fast evaluation of the resolution and noise properties of Bayesian reconstruction. The results have been used to choose the prior parameter to maximize the contrast to noise ratio $^{9}$ and to achieve uniform contrast recovery in fully 3D PET. ${ }^{10}$ Similar approximations have also been used by Stayman and Fessler ${ }^{11}$ in designing penalty function to achieve isotropic local point spread functions. Combining with computer observer models, these theoretical expressions have also been applied to the study of lesion detectability. ${ }^{14}$

Here we applied these results to the study of ROI quantitation. For quantitation, we are interested in bias and variance of the estimate. We will derive theoretical expressions for the bias and variance of ROI quantitation. From them we will construct the ensemble mean squared error (EMSE). One difficulty in evaluating ROI quantitation is that the truth is required for calculating the bias. We overcome this problem by using ensemble knowledge of the activity distribution inside the ROI. We will derive expression for the overall EMSE and show that the prior parameter can be selected to minimize the overall EMSE.

\section{THEORY}

\subsection{Data Model}

Emission data are well modeled as a collection of independent Poisson random variables with the expectation, $\overline{\boldsymbol{y}} \in \mathbb{R}^{M \times 1}$, related to the unknown tracer distribution, $\boldsymbol{x} \in \mathbb{R}^{N \times 1}$, through an affine transform

$$
\overline{\boldsymbol{y}}=\boldsymbol{P} \boldsymbol{x}+\boldsymbol{r},
$$

where $\boldsymbol{P} \in \mathbb{R}^{M \times N}$ is the detection probability matrix with the $(i, j)$ th element equal to the probability of detecting an event from the $j$ th voxel at the $i$ th measurement, and $\boldsymbol{r} \in \mathbb{R}^{M \times 1}$ accounts for the presence of scattered and random events in the data.

The Poisson likelihood function is

$$
p(\boldsymbol{y} \mid \boldsymbol{x})=\prod_{i} \frac{e^{-\bar{y}_{i}} \bar{y}_{i}^{y_{i}}}{y_{i} !}
$$

and the log likelihood function is given by

$$
L(\boldsymbol{y} \mid \boldsymbol{x})=\sum_{i}\left(y_{i} \log \bar{y}_{i}-\bar{y}_{i}-\log y_{i} !\right),
$$

where $\boldsymbol{y} \in \mathbb{R}^{M \times 1}$ is the measured sinogram data.

In PET the detection probability matrix $\boldsymbol{P}$ can be factored into

$$
\boldsymbol{P}=\boldsymbol{P}_{\text {sens }} \boldsymbol{P}_{\text {attn }} \boldsymbol{P}_{\text {sys }},
$$

where $\boldsymbol{P}_{\mathrm{sys}}$ is a projection matrix determined by the PET scanner geometry, with each element $(i, j)$ equal to the probability of a photon pair produced in voxel $j$ being detected by the detector pair $i$ in the absence of attenuation and assuming uniform detector sensitivity. $\boldsymbol{P}_{\text {attn }}$ and $\boldsymbol{P}_{\text {sens }}$ are diagonal matrices that account for the photon attenuation inside the object and the variation of the detector sensitivities, respectively. In SPECT the photon attenuation also depends on the source location and hence cannot be factored out from $\boldsymbol{P}_{\text {sys }}$.

\subsection{Bayesian Image Reconstruction}

The ML estimate can be found by maximizing (3). A popular ML algorithm for PET reconstruction is the EM algorithm. ${ }^{15-17}$ However, ML solutions are unstable (i.e., noisy) because the tomography problem is illconditioned. Thus, some form of regularization (or prior function) is needed to reconstruct a reasonable image. 
Bayesian methods regularize the image through the use of a prior distribution on the unknown image. Most image priors in emission image reconstruction have a Gibbs distribution of the form

$$
p(\boldsymbol{x})=\frac{1}{Z} e^{-\beta U(\boldsymbol{x})},
$$

where $U(\boldsymbol{x})$ is the energy function, $\beta$ is the smoothing parameter that controls the resolution of the reconstructed image, and $Z$ is a normalization constant. Here we focus on the widely used quadratic priors, for which the energy function can be expressed as

$$
U(\boldsymbol{x})=\frac{1}{2} \boldsymbol{x}^{\prime} \boldsymbol{R} \boldsymbol{x},
$$

where $\boldsymbol{R}$ is a positive definite (or semidefinite*) matrix. Pair-wise membrane priors ${ }^{18}$ and thin-plate priors ${ }^{18,19}$ are special cases of $(6)$.

Combining the likelihood function and the image prior, the MAP reconstruction is found as

$$
\hat{\boldsymbol{x}}(\boldsymbol{y})=\arg \max _{\boldsymbol{x} \geq 0}[L(\boldsymbol{y} \mid \boldsymbol{x})-\beta U(\boldsymbol{x})] .
$$

Since $L(\boldsymbol{y} \mid \boldsymbol{x})$ is a concave function of $\boldsymbol{x},(7)$ generally has a unique solution for convex priors. The smoothing parameter $\beta$ has a strong effect on the image property. If $\beta$ is too small, the reconstructed image approaches the ML estimate and becomes very noisy; if $\beta$ is too large, the reconstructed image will be very smooth and useful structural information can be lost. In the following sections we study how to select $\beta$ for the optimum ROI quantitation. We note that when $\beta$ is dependent on data, the method ceases to be true Bayesian. However, the same MAP reconstruction algorithm can still be used to reconstruct an image once $\beta$ is selected. Thus, with a little abuse of terminology, we still refer to the result as a MAP reconstruction.

\subsection{ROI Quantitation}

The total tracer uptake inside an ROI is computed as

$$
\eta(\hat{\boldsymbol{x}})=\boldsymbol{f}^{\prime} \hat{\boldsymbol{x}},
$$

where $\boldsymbol{f}$ is the indicator vector of the ROI, i.e., $f_{i}$ is equal to one if voxel $i$ is inside the ROI, and zero otherwise.

We are often interested in the bias and variance of the estimate,

$$
\begin{aligned}
\operatorname{bias}(\eta) & =\boldsymbol{f}^{\prime} \mathcal{E} \boldsymbol{y}\{\hat{\boldsymbol{x}}(\boldsymbol{y})\}-\boldsymbol{f}^{\prime} \boldsymbol{x}=\boldsymbol{f}^{\prime}[h(\boldsymbol{x})-\boldsymbol{x}], \\
\operatorname{var}(\eta) & =\boldsymbol{f}^{\prime} \Sigma_{\hat{\boldsymbol{x}}} \boldsymbol{f} .
\end{aligned}
$$

where $h(\boldsymbol{x})=\mathcal{E} \boldsymbol{y}(\hat{\boldsymbol{x}})$ is the expectation of the reconstructed images of the unknown tracer distribution $\boldsymbol{x}$ and $\Sigma_{\hat{\boldsymbol{x}}}$ is the covariance matrix of the reconstructed images.

In order to calculate the bias, we focus on small ROIs that are surrounded by some uniform background. Let $\boldsymbol{x}_{0}$ denote a reference image where the activity inside the ROI is equal to the uniform background and $h\left(\boldsymbol{x}_{0}\right)$ the expectation of the corresponding reconstructed image. If the size of the uniform background in $\boldsymbol{x}_{0}$ is much larger compared to the resolution of the reconstructed image, we can have the following approximation

$$
\boldsymbol{f}^{\prime} h\left(\boldsymbol{x}_{0}\right)-\boldsymbol{f}^{\prime} \boldsymbol{x}_{0} \approx 0 .
$$

Then (9) can be converted to

$$
\begin{aligned}
\operatorname{bias}\left(\eta_{\mathrm{ROI}}\right) & \approx \boldsymbol{f}^{\prime} h(\boldsymbol{x})-\boldsymbol{f}^{\prime} \boldsymbol{x}+\boldsymbol{f}^{\prime} \boldsymbol{x}_{0}-\boldsymbol{f}^{\prime} h\left(\boldsymbol{x}_{0}\right) \\
& =\boldsymbol{f}^{\prime}\left[h(\boldsymbol{x})-h\left(\boldsymbol{x}_{0}\right)\right]-\boldsymbol{f}^{\prime}\left[\boldsymbol{x}-\boldsymbol{x}_{0}\right] .
\end{aligned}
$$

* Strictly speaking, when $\boldsymbol{R}$ is semidefinite, the normalization constant $Z=\infty$. However, the MAP reconstruction in (7) is still well defined. 
Using the first-order Taylor series expansion on $h(\boldsymbol{x})$, we have

$$
h(\boldsymbol{x})=h\left(\boldsymbol{x}_{0}\right)+\nabla h\left(\boldsymbol{x}_{0}\right)\left(\boldsymbol{x}-\boldsymbol{x}_{0}\right) .
$$

For MAP reconstruction shown in (7) with the Poisson likelihood, we can derive ${ }^{7}$

$$
\nabla h(\boldsymbol{x})=[\boldsymbol{F}+\beta \boldsymbol{R}]^{-1} \boldsymbol{F},
$$

where $\boldsymbol{F}=\boldsymbol{P}^{\prime} \operatorname{diag}\left[\frac{1}{\bar{y}_{i}}\right] \boldsymbol{P}$ is the Fisher information matrix.

Substituting (13) and (14) into (12) results in

$$
\operatorname{bias}(\eta) \approx \boldsymbol{f}^{\prime}[\boldsymbol{F}+\beta \boldsymbol{R}]^{-1} \boldsymbol{F} \boldsymbol{x}_{\mathrm{ROI}}-\boldsymbol{f}^{\prime} \boldsymbol{x}_{\mathrm{ROI}},
$$

where $\boldsymbol{x}_{\mathrm{ROI}}=\boldsymbol{x}-\boldsymbol{x}_{0}$ is the activity inside the ROI above the background.

Similarly, using the first-order Taylor series expansion on $\hat{\boldsymbol{x}}(\boldsymbol{y})$, we can derive an approximation for the covariance matrix ${ }^{6}$

$$
\Sigma_{\hat{x}} \approx[\boldsymbol{F}+\beta \boldsymbol{R}]^{-1} \boldsymbol{F}[\boldsymbol{F}+\beta \boldsymbol{R}]^{-1} .
$$

Thus, the variance of $\eta$ is

$$
\operatorname{var}(\eta)=\boldsymbol{f}^{\prime}[\boldsymbol{F}+\beta \boldsymbol{R}]^{-1} \boldsymbol{F}[\boldsymbol{F}+\beta \boldsymbol{R}]^{-1} \boldsymbol{f} .
$$

Combining the bias and variance, we can obtain a single figure of merit for measuring the quality of the quantitation, the ensemble mean squared error (EMSE),

$$
\begin{aligned}
\mathrm{EMSE} & =[\operatorname{bias}(\eta)]^{2}+\operatorname{var}(\eta) \\
& =\left|\boldsymbol{f}^{\prime}[\boldsymbol{F}+\beta \boldsymbol{R}]^{-1} \boldsymbol{F} \boldsymbol{x}_{\mathrm{ROI}}-\boldsymbol{f}^{\prime} \boldsymbol{x}_{\mathrm{ROI}}\right|^{2}+\boldsymbol{f}^{\prime}[\boldsymbol{F}+\beta \boldsymbol{R}]^{-1} \boldsymbol{F}[\boldsymbol{F}+\beta \boldsymbol{R}]^{-1} \boldsymbol{f} .
\end{aligned}
$$

\subsection{ROI with Random Activity}

Equation (18) is not computable in real situations since we do not know $\boldsymbol{x}_{\mathrm{ROI}}$ (otherwise the ROI quantitation would not be necessary). However, we often have some knowledge of the statistical distribution of the ROI activity based on existing data. We can then study the averaged ensemble mean squared error for all possible ROI activity

$$
\overline{\mathrm{EMSE}}=\mathcal{E}\left(\eta-\boldsymbol{f}^{\prime} \boldsymbol{x}\right)^{2}=\mathcal{E}_{\mathrm{ROI}}\left[\operatorname{bias}\left(\eta \mid \boldsymbol{x}_{\mathrm{ROI}}\right)\right]^{2}+\mathcal{E}_{\mathrm{ROI}} \operatorname{var}\left(\eta \mid \boldsymbol{x}_{\mathrm{ROI}}\right),
$$

where $\mathcal{E}_{\text {ROI }}$ denotes the expectation w.r.t. the distribution of the ROI activity, and bias $\left(\eta \mid \boldsymbol{x}_{\mathrm{ROI}}\right)$ and $\operatorname{var}\left(\eta \mid \boldsymbol{x}_{\mathrm{ROI}}\right)$ denote the bias and variance of the ROI quantitation with known ROI activity as shown in (15) and (17), respectively. Please note that $\boldsymbol{x}$ in (19) is a random vector.

To simplify the notations, let us define $\boldsymbol{q} \equiv \boldsymbol{F}[\boldsymbol{F}+\beta \boldsymbol{R}]^{-1} \boldsymbol{f}$. From (15) we have

$$
\begin{aligned}
\mathcal{E}_{\mathrm{ROI}}\left[\operatorname{bias}\left(\eta \mid \boldsymbol{x}_{\mathrm{ROI}}\right)\right]^{2} & \approx \mathcal{E}_{\mathrm{ROI}}(\boldsymbol{q}-\boldsymbol{f})^{\prime} \boldsymbol{x}_{\mathrm{ROI}} \boldsymbol{x}_{\mathrm{ROI}}^{\prime}(\boldsymbol{q}-\boldsymbol{f}), \\
& \approx(\boldsymbol{q}-\boldsymbol{f})^{\prime} \mathcal{E}_{\mathrm{ROI}}\left\{\boldsymbol{x}_{\mathrm{ROI}} \boldsymbol{x}_{\mathrm{ROI}}^{\prime}\right\}(\boldsymbol{q}-\boldsymbol{f}), \\
& \approx(\boldsymbol{q}-\boldsymbol{f})^{\prime} \Sigma_{\mathrm{ROI}}(\boldsymbol{q}-\boldsymbol{f})^{\prime}+\left|(\boldsymbol{q}-\boldsymbol{f})^{\prime} \overline{\boldsymbol{x}}_{\mathrm{ROI}}\right|^{2},
\end{aligned}
$$

where $\Sigma_{\mathrm{ROI}}$ and $\overline{\boldsymbol{x}}_{\mathrm{ROI}}$ are the covariance and the expectation of the ROI activity.

Now let us study the expectation of the variance. Since the ROI is small compared to the whole object, we can assume that the variation of the activity in the ROI is insignificant compared to the Poisson noise in the emission data. Thus, the covariance of the reconstructed image in (16) is independent of the ROI activity, i.e.,

$$
\mathcal{E}_{\mathrm{ROI}} \operatorname{var}\left(\eta \mid \boldsymbol{x}_{\mathrm{ROI}}\right)=\operatorname{var}\left(\eta \mid \boldsymbol{x}_{\mathrm{ROI}}\right) \approx \boldsymbol{q}^{\prime}[\boldsymbol{F}+\beta \boldsymbol{R}]^{-1} \boldsymbol{f} .
$$

Substituting (22) and (23) into (19), we get

$$
\overline{\mathrm{EMSE}} \approx(\boldsymbol{q}-\boldsymbol{f})^{\prime} \Sigma_{\mathrm{ROI}}(\boldsymbol{q}-\boldsymbol{f})^{\prime}+\left|(\boldsymbol{q}-\boldsymbol{f})^{\prime} \overline{\boldsymbol{x}}_{\mathrm{ROI}}\right|^{2}+\boldsymbol{q}^{\prime}[\boldsymbol{F}+\beta \boldsymbol{R}]^{-1} \boldsymbol{f} .
$$


On the other hand, we can write

$$
\overline{\mathrm{EMSE}}=\mathcal{E}\left(\eta-\boldsymbol{f}^{\prime} \boldsymbol{x}\right)^{2}=\left[\mathcal{E}\left(\eta-\boldsymbol{f}^{\prime} \boldsymbol{x}\right)\right]^{2}+\operatorname{var}\left(\eta-\boldsymbol{f}^{\prime} \boldsymbol{x}\right) .
$$

Comparing (24) and (25), we have

$$
\begin{aligned}
\mathcal{E}\left(\eta-\boldsymbol{f}^{\prime} \boldsymbol{x}\right) & \approx(\boldsymbol{q}-\boldsymbol{f})^{\prime} \overline{\boldsymbol{x}}_{\mathrm{ROI}} \\
\operatorname{var}\left(\eta-\boldsymbol{f}^{\prime} \boldsymbol{x}\right) & \approx \boldsymbol{q}^{\prime}[\boldsymbol{F}+\beta \boldsymbol{R}]^{-1} \boldsymbol{f}+(\boldsymbol{q}-\boldsymbol{f})^{\prime} \Sigma_{\mathrm{ROI}}(\boldsymbol{q}-\boldsymbol{f})^{\prime} .
\end{aligned}
$$

The first term in (27) is the variance caused by the Poisson noise, while the second term is caused by the variation of ROI activity.

\subsection{ROI with Variable Contrast}

As a special case, we study the ROI with variable contrast in this section. Here the activity distribution in the ROI is modeled as a fixed profile times a random scaling factor (variable contrast), i.e.,

$$
\boldsymbol{x}_{\mathrm{ROI}}=c \overline{\boldsymbol{x}}_{\mathrm{ROI}},
$$

where $c$ is the random scaling factor with mean of one and variance of $\sigma_{c}^{2}$. This statistical model is very useful in emission imaging because the tracer uptake in many ROIs can be assumed to be homogeneous. The variance of $\boldsymbol{x}_{\mathrm{ROI}}$ under this model is

$$
\Sigma_{\mathrm{ROI}}=\sigma_{c}^{2} \overline{\boldsymbol{x}}_{\mathrm{ROI}} \overline{\boldsymbol{x}}_{\mathrm{ROI}}^{\prime}
$$

Substituting it into (24) results in

$$
\overline{\mathrm{EMSE}}=\left(1+\sigma_{c}^{2}\right)\left|(\boldsymbol{q}-\boldsymbol{f})^{\prime} \overline{\boldsymbol{x}}_{\mathrm{ROI}}\right|^{2}+\boldsymbol{q}^{\prime}[\boldsymbol{F}+\beta \boldsymbol{R}]^{-1} \boldsymbol{f} .
$$

Equation (30) is equivalent to a generalized ensemble mean squared error of the ROI quantitation with known activity by giving a weight of $\left(1+\sigma_{c}^{2}\right)$ to the bias square.

\subsection{Fast Computation}

Equations (24) and (30) involve the inverse of $[\boldsymbol{F}+\beta \boldsymbol{R}]$. Direct calculation is very time-consuming due to the large size of the matrix. Here we derive simplified expressions using Fourier transform. The idea is based on the observation that $\boldsymbol{F}$ and $\boldsymbol{F}+\beta \boldsymbol{R}$ are diagonally dominant and are smoothly varying along the diagonal. ${ }^{6,7}$ This allows us to use the locally stationary approximation ${ }^{10}$

$$
\boldsymbol{q} \approx \boldsymbol{F}(j)[\boldsymbol{F}(j)+\beta \boldsymbol{R}(j)]^{-1} \boldsymbol{f}
$$

where $\boldsymbol{F}(j)$ and $\boldsymbol{R}(j)$ are block Toeplitz matrices whose $j$ th columns are equal to the $j$ th columns of $\boldsymbol{F}$ and $\boldsymbol{R}$, respectively (assuming voxel $j$ is the center of the ROI).

Since a large block Toeplitz matrix can be approximately diagonalized by Fourier transform, (31) can be computed as

$$
\begin{aligned}
\boldsymbol{q} & \approx \boldsymbol{Q}^{\prime} \boldsymbol{Q} \boldsymbol{F}(j) \boldsymbol{Q}^{\prime} \boldsymbol{Q}[\boldsymbol{F}(j)+\beta \boldsymbol{R}(j)]^{-1} \boldsymbol{Q}^{\prime} \boldsymbol{Q} \boldsymbol{f} \\
& \approx \boldsymbol{Q}^{\prime} \operatorname{diag}\left[\frac{\lambda_{i}(j)}{\lambda_{i}(j)+\beta \mu_{i}(j)}\right] \boldsymbol{Q} \boldsymbol{f}
\end{aligned}
$$

where $\left\{\lambda_{i}(j), i=1, \ldots, N\right\}$ is the Fourier transform of $\boldsymbol{F}(j)$ and $\left\{\mu_{i}(j), i=1, \ldots, N\right\}$ is the Fourier transform of $\boldsymbol{R}(j) . \boldsymbol{Q}$ and $\boldsymbol{Q}^{\prime}$ represent the Kronecker form of the Fourier transform and its inverse, respectively. Details on the computation of $\boldsymbol{\lambda}(j)$ and $\boldsymbol{\mu}(j)$ can be found in. ${ }^{10}$

Similarly, $[\boldsymbol{F}+\beta \boldsymbol{R}]^{-1} \boldsymbol{f}$ can be computed as

$$
[\boldsymbol{F}+\beta \boldsymbol{R}]^{-1} \boldsymbol{f} \approx \boldsymbol{Q}^{\prime} \operatorname{diag}\left[\frac{1}{\lambda_{i}(j)+\beta \mu_{i}(j)}\right] \boldsymbol{Q} \boldsymbol{f} .
$$


Using the above approximation, (30) becomes

$$
\begin{aligned}
\overline{\mathrm{EMSE}} \approx & \left(1+\sigma_{c}^{2}\right)\left(\boldsymbol{f}^{\prime} \boldsymbol{Q}^{\prime} \operatorname{diag}\left[\frac{\lambda_{i}(j)}{\lambda_{i}(j)+\beta \mu_{i}(j)}\right] \boldsymbol{Q} \overline{\boldsymbol{x}}_{\mathrm{ROI}}-\boldsymbol{f}^{\prime} \overline{\boldsymbol{x}}_{\mathrm{ROI}}\right)^{2} \\
& +\boldsymbol{f}^{\prime} \boldsymbol{Q}^{\prime} \operatorname{diag}\left[\frac{\lambda_{i}(j)}{\left(\lambda_{i}(j)+\beta \mu_{i}(j)\right)^{2}}\right] \boldsymbol{Q} \boldsymbol{f} \\
= & \left(1+\sigma_{c}^{2}\right)\left(\sum_{i} \frac{\lambda_{i}(j) \xi_{i}^{*} \zeta_{i}}{\lambda_{i}(j)+\beta \mu_{i}(j)}-\boldsymbol{f}^{\prime} \overline{\boldsymbol{x}}_{\mathrm{ROI}}\right)^{2}+\sum_{i} \frac{\lambda_{i}(j)\left|\xi_{i}\right|^{2}}{\left(\lambda_{i}(j)+\beta \mu_{i}(j)\right)^{2}},
\end{aligned}
$$

where $\left\{\xi_{i}, i=1, \ldots, N\right\}$ and $\left\{\zeta_{i}, i=1, \ldots, N\right\}$ are the Fourier transforms of $\boldsymbol{f}$ and $\overline{\boldsymbol{x}}_{\mathrm{ROI}}$, respectively, and * denotes the conjugate operation.

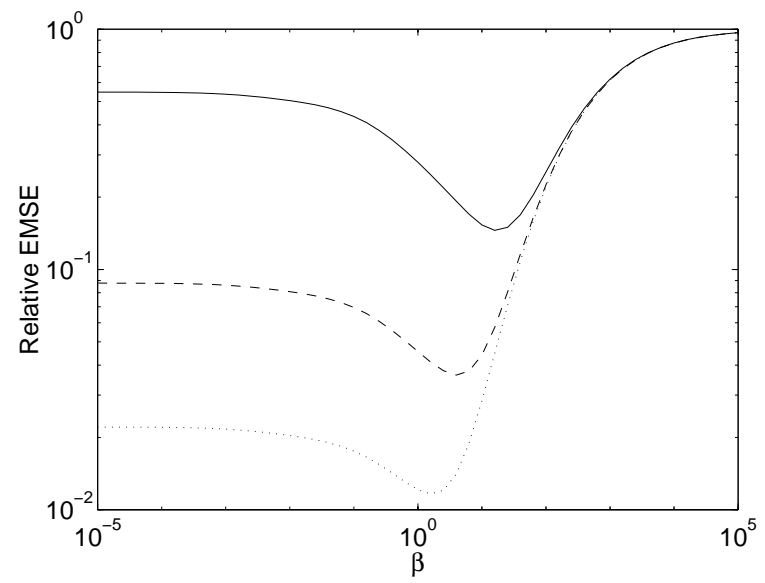

(a)

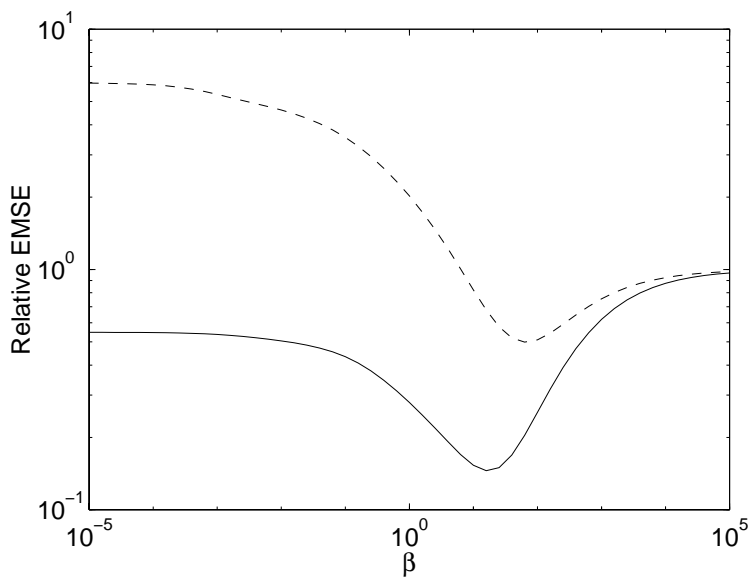

(b)

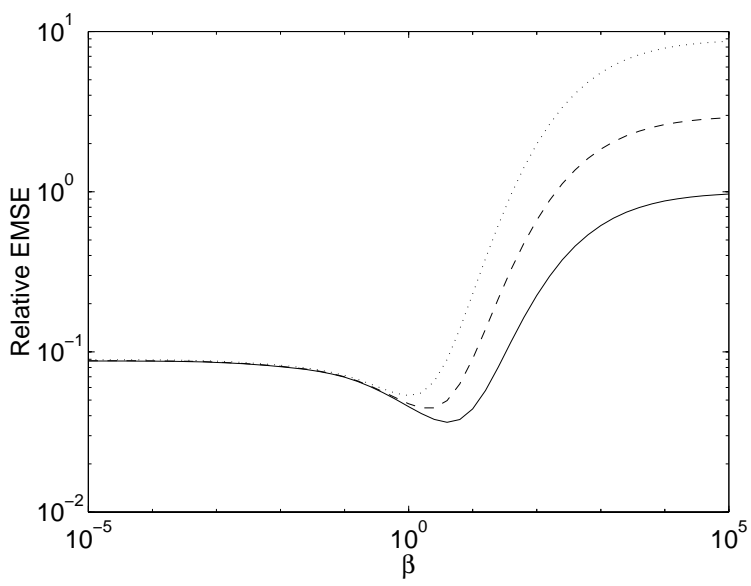

(c)

Figure 1. Plots of relative EMSE of the ROI quantitation as a function of $\beta$ for different ROIs. (a) ROIs (diameter $=$ $16 \mathrm{~mm}$ ) with different mean activity: 2 (solid line), 5 (dashed line), and 10 (dotted line). (b) ROIs (mean activity $=2$ ) of different diameter: $16 \mathrm{~mm}$ (solid line) and $8 \mathrm{~mm}$ (dashed line). There is no variability of the ROI activity in both (a) and $(\mathrm{b})$. $(\mathrm{c}) \mathrm{ROIs}\left(\right.$ diameter $=16 \mathrm{~mm}$, mean activity $=5$ ) with variable contrast: $\sigma_{c}^{2}=0$ (solid line), $\sigma_{c}^{2}=2$ (dashed line), and $\sigma_{c}^{2}=10$ (dotted line).

Equation (35) allows fast evaluation of average EMSE. To achieve the minimum EMSE estimate of the ROI uptake, we can evaluate (35) for different $\beta$ and choose the $\beta$ that gives the minimum value. Figure 1 shows the 
plots of the relative EMSE $\left(=\overline{\mathrm{EMSE}} /\left|\boldsymbol{f}^{\prime} \overline{\boldsymbol{x}}_{\mathrm{ROI}}\right|^{2}\right)$ as a function of the smoothing parameter $\beta$ for different ROI activity. It shows that the EMSE not only depends on the smoothing parameter $\beta$, but also depends on the size, activity level, and variability of the ROI. As the mean activity or size increases, the relative EMSE decreases, and so does the optimum smoothing parameter. When the variation of the ROI activity increases, the relative EMSE increases, but the optimum smoothing parameter decreases.

These plots show that the optimum smoothing parameter highly depends on the ROI activity. The ensemble mean and covariance of the ROI activity is essential in the selection of the optimum smoothing parameter. However, we can also see that for ROIs with high total activity, the EMSE with very small $\beta$ is close to the minimum; while for ROIs with low total activity, the EMSE with large $\beta$ is close the minimum. This can give us some rough guidance when the statistical knowledge of the ROI activity is uncertain.

\section{SIMULATION RESULTS}

We conducted computer simulations to validate our theoretical approximations. We simulated an ECAT HR+ whole-body PET scanner (CTI PET Systems, Knoxville, TN) operating in two dimension mode. The scanner has 576 detectors forming a ring with a $413 \mathrm{~mm}$ radius. The sinogram data has 288 angles of view and 288 lines of response in each view. The background phantom image was obtained from a reconstructed patient scan (Figure 2a). Two lesions, one $8 \mathrm{~mm}$ diameter and one $16 \mathrm{~mm}$ diameter, were simulated in the liver region as shown in Figure 2a. ROIs were selected for both lesions. The attenuation factors that were used in the simulation were obtained from the same patient data (Figure $2 \mathrm{~b}$ ).

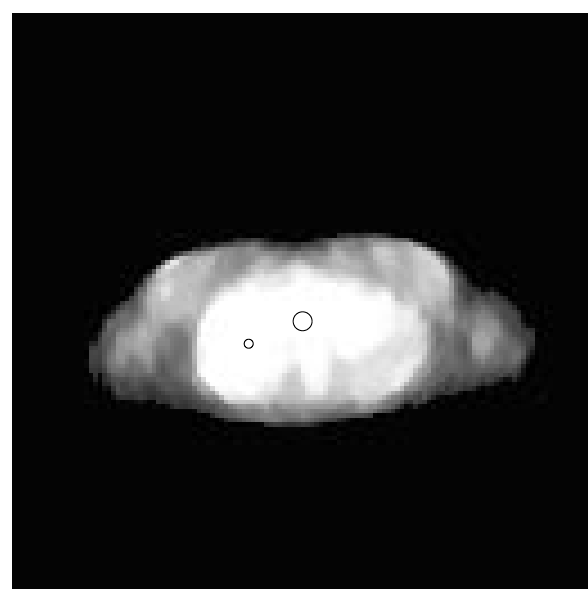

(a)
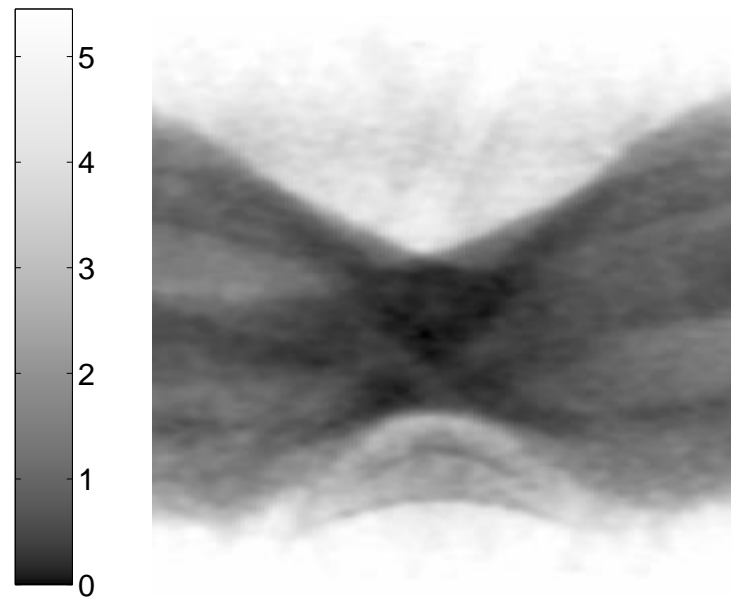

(b)

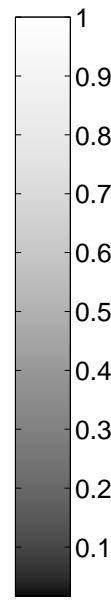

0.1

Figure 2. The background phantom image (a) and the corresponding attenuation factors (b).

The data were generated by forward projecting the phantom image with attenuation. Poisson noises were added to the sinogram data after scaling the expected total number of events to 200k. The noisy data sets were reconstructed using a preconditioned conjugated gradient method. ${ }^{20}$ The activities in the two selected ROIs were measured for each reconstructed image, from which the EMSE was calculated.

Figure 3 shows the comparison of the EMSE from 300 independent Monte Carlo reconstructions and the theoretical approximation without ROI activity variation. Figure 4 shows the comparison of the EMSE from 200 independent Monte Carlo reconstructions and the theoretical approximation for ROIs with variable contrast. The standard deviations of the Monte Carlo results were estimated using a bootstrap method. The theoretical approximation was compensated for the non-negativity constraint using the method developed by Qi and Leahy ${ }^{10}$ with a low-resolution noisy reconstruction $(\beta=100)$. Overall, the theoretical results provide good predictions on the minimum EMSE and the optimum smoothing parameter for both ROIs. 


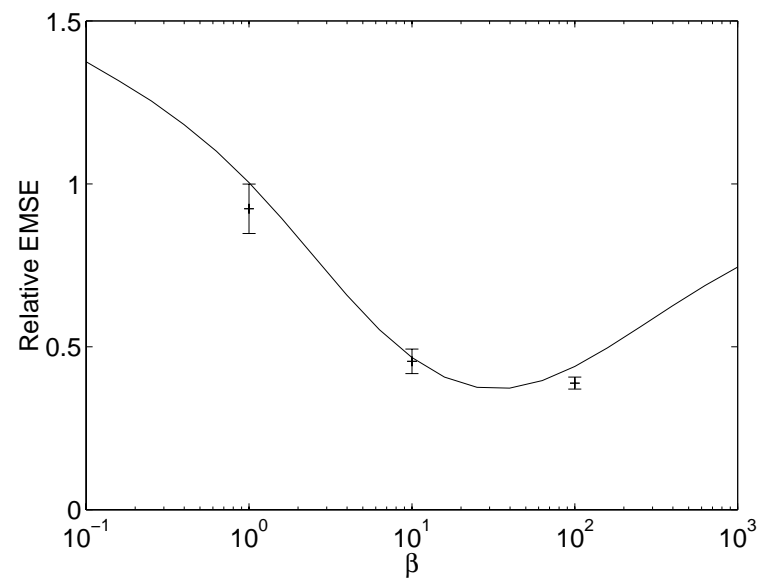

(a)

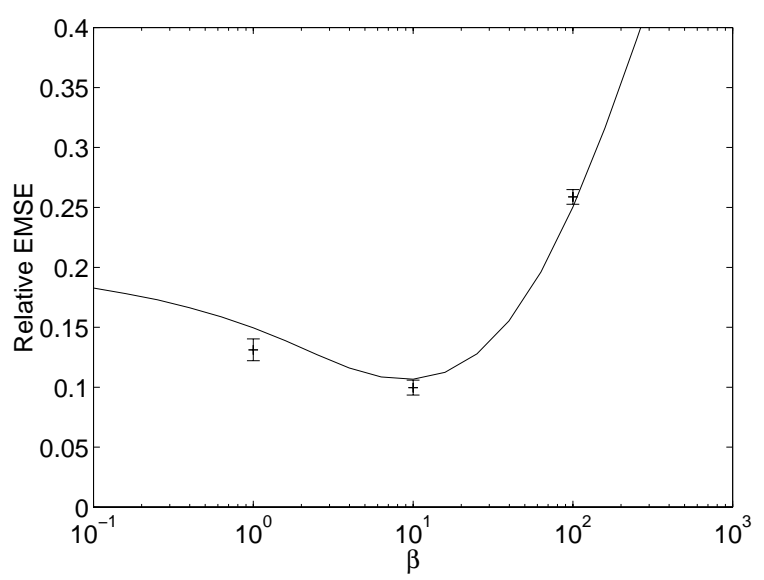

(b)

Figure 3. Comparison of the Monte Carlo results ('+') with the theoretical approximations (solid line). (a) An 8mm diameter ROI; (b) a $16 \mathrm{~mm}$ diameter ROI. The mean activity in both ROIs is about 2.6, and there is no variation in ROI activity. The error bars represent plus and minus one standard deviation.

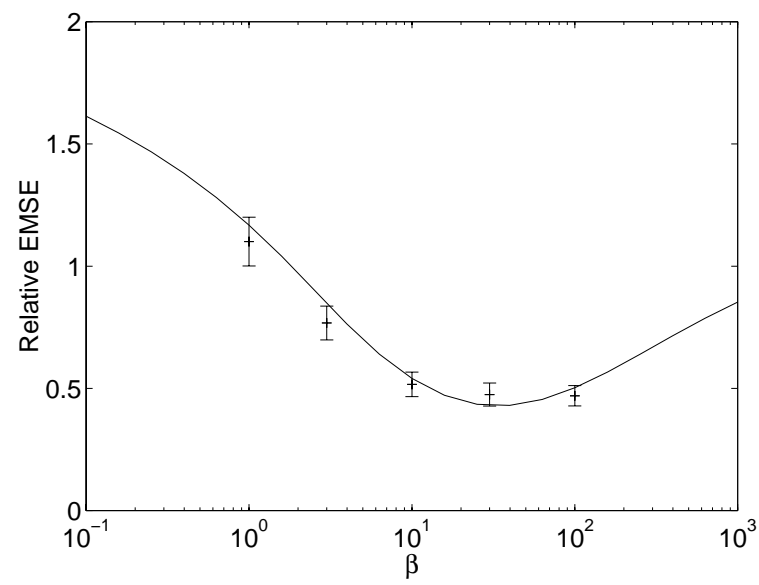

(a)

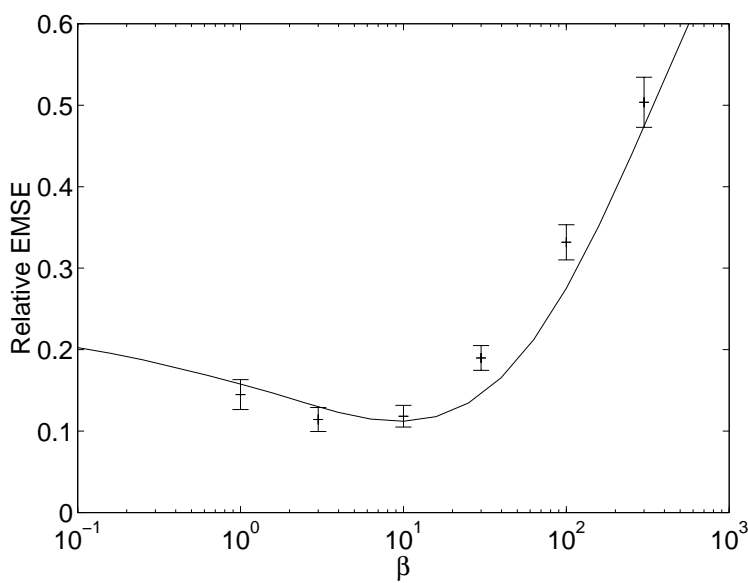

(b)

Figure 4. Comparison of the Monte Carlo results ('+') with the theoretical approximations (solid line). (a) An 8mm diameter ROI; (b) a 16mm diameter ROI. The activities in both ROIs are uniformly distributed between 1 to 4 .

\section{CONCLUSION AND DISCUSSION}

We have presented a theoretical analysis of ROI quantitation for the Bayesian reconstruction. Simplified expressions for the bias, variance and ensemble mean squared error for ROI quantitation have been derived. These expressions allow fast evaluation of image quality and can be used to select the optimum smoothing parameter to achieve the minimum EMSE estimate of the ROI activity.

While we have been emphasized on minimizing the EMSE, the results can also be applied to other figures of merit, such as the generalized EMSE (weighted sum of bias square and variance). For experiments that are more tolerant to bias (or variance), one can assign less weight to the bias square (or variance) to achieve a better result. The smoothing parameter that minimizes the generalized EMSE can be found using a similar approach.

We have shown that the optimum smoothing parameter depends on the size and activity distribution of the ROI. As a result the ROI has to be defined before selecting the optimum smoothing parameter. This can be done 
by either drawing the ROI on an initial reconstruction or drawing the ROI on a co-registered anatomical image. With the recent development of combined PET/CT and SPECT/CT scanners, such high-resolution anatomical images are readily available. In emission tomography the tracer uptake can often be assumed to be homogeneous inside a small ROI. This allows the use of the variable contrast model. We have found that with this model we can apply corrections to the ROI estimate to further reduce the EMSE. We plan to study this issue in future work.

\section{ACKNOWLEDGMENTS}

The author would like to thank Robert Smith of Lawrence Berkeley National Laboratory for his copyediting.

This work is supported in part by the U.S. Department of Health and Human Services, National Heart, Lung and Blood Institute under grant P01 HL25840; by the National Institute of Biomedical Imaging and Bioengineering under grant R01 EB00363; and by the Director, Office of Science, Office of Biological and Environmental Research, Medical Sciences Division, of the U.S. Department of Energy under contract no. DE-AC03-76SF00098.

\section{REFERENCES}

1. J. A. Fessler, "Penalized weighted least squares image reconstruction for PET," IEEE Transactions on Medical Imaging 13, pp. 290-300, 1994.

2. E. Mumcuoglu, R. Leahy, S. Cherry, and Z. Zhou, "Fast gradient-based methods for Bayesian reconstruction of transmission and emission PET images," IEEE Transactions on Medical Imaging 13, pp. 687-701, December 1994.

3. J. A. Fessler and A. O. Hero, "Penalized maximum-likelihood image reconstruction using space-alternating generalized EM algorithms," IEEE Transactions on Image Processing 4, pp. 1417-1429, Oct 1995.

4. C. Bouman and K. Sauer, "A unified approach to statistical tomography using coordinate descent optimization," IEEE Transactions on Image Processing 5, pp. 480-492, March 1996.

5. H. H. Barrett, D. W. Wilson, and B. M. W. Tsui, "Noise properties of the EM algorithm: I. theory," Physics in Medicine and Biology 39, pp. 833-846, 1994.

6. J. A. Fessler, "Mean and variance of implicitly defined biased estimators (such as penalized maximum likelihood): Applications to tomography," IEEE Transactions on Image Processing 5, pp. 493-506, March 1996.

7. J. A. Fessler and W. L. Rogers, "Spatial resolution properties of penalized-likelihood image reconstruction: Spatial-invariant tomographs," IEEE Transactions on Image Processing 9, pp. 1346-1358, September 1996.

8. W. Wang and G. Gindi, "Noise analysis of MAP-EM algorithms for emission tomography," Physics in Medicine and Biology 42, pp. 2215-2232, 1997.

9. J. Qi and R. M. Leahy, "A theoretical study of the contrast recovery and variance of MAP reconstructions from PET data," IEEE Transactions on Medical Imaging 18(4), pp. 293-305, 1999.

10. J. Qi and R. M. Leahy, "Resolution and noise properties of MAP reconstruction for fully 3D PET," IEEE Transactions on Medical Imaging 19(5), pp. 493-506, 2000.

11. J. W. Stayman and J. A. Fessler, "Regularization for uniform spatial resolution properties in penalizedlikelihood image reconstruction," IEEE Transactions on Medical Imaging 19, pp. 601-615, 2000.

12. E. J. Soares, C. L. Byrne, and S. J. Glick, "Noise characterization of block-iterative reconstruction algorithms: I. theory," IEEE Transactions on Medical Imaging 19(4), pp. 261-270, 2000.

13. H. M. Hudson and R. S. Larkin, "Accelerated image reconstruction using ordered subsets of projection data," IEEE Transactions on Medical Imaging 13(4), pp. 601-609, 1994.

14. J. Qi and R. H. Huesman, "Theoretical study of lesion detectability of MAP reconstruction using computer observers," IEEE Transactions on Medical Imaging 20, pp. 815-822, August 2001.

15. A. Dempster, N. Laird, and D. Rubin, "Maximum likelihood from incomplete data via the EM algorithm," Journal of Royal Statistical Society, Series B 39(1), pp. 1-38, 1977.

16. L. Shepp and Y. Vardi, "Maximum likelihood reconstruction for emission tomography," IEEE Transactions on Medical Imaging 1, pp. 113-122, October 1982. 
17. K. Lange and R. Carson, "EM reconstruction algorithms for emission and transmission tomography," Journal of Computer Assisted Tomography 8, pp. 306-316, April 1984.

18. A. Blake and A. Zisserman, Visual Reconstruction, The MIT Press, 1987.

19. S.-J. Lee, A. Rangarajan, and G. Gindi, "Bayesian image reconstruction in SPECT using higher oreder mechanical models as priors," IEEE Transactions on Medical Imaging 14, pp. 669-680, December 1995.

20. J. Qi, R. M. Leahy, S. R. Cherry, A. Chatziioannou, and T. H. Farquhar, "High resolution 3D Bayesian image reconstruction using the microPET small animal scanner," Physics in Medicine and Biology 43(4), pp. 1001-1013, 1998. 